\title{
SHARP UNCERTAINTY PRINCIPLES ON RIEMANNIAN MANIFOLDS: THE INFLUENCE OF CURVATURE
}

\author{
ALEXANDRU KRISTÁLY
}

\begin{abstract}
We present a rigidity scenario for complete Riemannian manifolds supporting the Heisenberg-Pauli-Weyl uncertainty principle with the sharp constant in $\mathbb{R}^{n}$ (shortly, sharp HPW principle). Our results deeply depend on the curvature of the Riemannian manifold which can be roughly formulated as follows:

(a) When $(M, g)$ has non-positive sectional curvature, the sharp HPW principle holds on $(M, g)$. However, positive extremals exist in the sharp HPW principle if and only if $(M, g)$ is isometric to $\mathbb{R}^{n}, n=\operatorname{dim}(M)$.

(b) When $(M, g)$ has non-negative Ricci curvature, the sharp HPW principle holds on $(M, g)$ if and only if $(M, g)$ is isometric to $\mathbb{R}^{n}$.

Since the sharp HPW principle and the Hardy-Poincaré inequality are endpoints of the Caffarelli-Kohn-Nirenberg interpolation inequality, we establish further quantitative results for the latter inequalities in terms of the curvature on Cartan-Hadamard manifolds.

Nous présentons un scénario de rigidité pour les variétés riemanniennes complètes soutenant le principe d'incertitude d'Heisenberg-Pauli-Weyl avec la constante optimale en $\mathbb{R}^{n}$ (brièvement, le principle d'HPW). Nos rsultats dpendent profondment de la courbure de la variété riemannienne et ils peuvent être formulés comme suit :

(a) Lorsque $(M, g)$ a courbure sectionnelle non positive, le principe d'HPW (se maintient) a lieu sur $(M, g)$. Néanmoins, des fonctions extrémales positives existent dans le principe d'HPW si et seulement si $(M, g)$ est isométrique à $\mathbb{R}^{n}, n=\operatorname{rmdim}(M)$.

(b) Lorsque $(M, g)$ a courbure de Ricci non ngative, le principe d'HPW a lieu sur $(M, g)$ si et seulement si $(M, g)$ est isométrique à $\mathbb{R}^{n}$.

Comme le principe d'HPW et l'ingalité Hardy-Poincaré sont des cas extrêmes de l'inégalité d'interpolation de Caffarelli-Kohn-Nirenberg, nous établissons des résultats quantitatifs pour les dernières inégalités en terme de la courbure sur les variétés de Cartan-Hadamard.
\end{abstract}

Dedicated to professor Zoltán M. Balogh on the occasion of his 50th birthday

\section{INTRODUCTION AND MAIN RESULTS}

The Heisenberg uncertainty principle in quantum mechanics states that the position and momentum of a given particle cannot be accurately determined simultaneously, see [25]. The rigorous mathematical formulation of this principle is attributed to Pauli and Weyl [37], stating that the function itself and its Fourier transform cannot be sharply localized at the same time. In terms of PDEs, the Heisenberg-Pauli-Weyl uncertainty principle in the Euclidean setting is described by the inequality

$$
\left(\int_{\mathbb{R}^{n}}|\nabla u(x)|^{2} \mathrm{~d} x\right)\left(\int_{\mathbb{R}^{n}}|x|^{2} u(x)^{2} \mathrm{~d} x\right) \geq \frac{n^{2}}{4}\left(\int_{\mathbb{R}^{n}} u(x)^{2} \mathrm{~d} x\right)^{2}, \forall u \in C_{0}^{\infty}\left(\mathbb{R}^{n}\right) .
$$

2000 Mathematics Subject Classification. Primary 53C21; Secondary 58J60.

Key words and phrases. Heisenberg-Pauli-Weyl uncertainty principle; Riemannian manifold; sharp constant; curvature; rigidity.

Research supported by the Project CNFIS-FDI-2016-0056, STAR-UBB Fellowship. 
It is well known that the constant $\frac{n^{2}}{4}$ is sharp and the extremals are given (up to a constant) by the family of Gaussian functions $u_{\lambda}(x)=e^{-\lambda|x|^{2}}, \lambda>0$.

Since its initial formulation, the Heisenberg-Pauli-Weyl principle is deserving continuously a deep source of inspiration in different areas of Physics and Mathematics. Without the sake of completeness, Heisenberg-Pauli-Weyl principle has been studied in various contexts, see Ciatti, Ricci and Sundari [13] (for positive self-adjoint operators on measure spaces), Fefferman [19], Folland and Sitaram [21], and Nahmod [33] (locating eigenvalues for selfadjoint differential operators via SAK principle), Andersen [2, 3], Erb [17, 18] and Kombe and Özaydin [28, 29] (sharp uncertainty principle on compact/noncompact Riemannian manifolds), Okoudjou, Saloff-Coste and Teplyaev [34] (for fractals, graphs and metric measure spaces), and references therein.

The purpose of our paper is to describe a complete scenario concerning the sharp Heisenberg-Pauli-Weyl uncertainty principle on complete Riemannian manifolds. Hereafter, in order to avoid confusions, the sharpness is understood in the sense that the HeisenbergPauli-Weyl principle holds on a Riemannian manifold $(M, g)$ with the same constant $\frac{n^{2}}{4}$ as in the Euclidean space $\mathbb{R}^{n}$.

To be more precise, let $(M, g)$ be an $n(\geq 2)$-dimensional complete Riemannian manifold, its canonical volume element $\mathrm{d} V_{g}$, and $d_{x_{0}}(x)=d\left(x_{0}, x\right)$ be the distance function from a point $x_{0} \in M$. For $x_{0} \in M$ fixed, we consider the Heisenberg-Pauli-Weyl principle on $(M, g)$ of the form: for all $u \in C_{0}^{\infty}(M)$,

$$
\left(\int_{M}\left|\nabla_{g} u\right|^{2} \mathrm{~d} V_{g}\right)\left(\int_{M} d_{x_{0}}^{2} u^{2} \mathrm{~d} V_{g}\right) \geq \frac{n^{2}}{4}\left(\int_{M} u^{2} \mathrm{~d} V_{g}\right)^{2} .
$$

$(\mathbf{H P W})_{x_{0}}$

Our first result can be stated as follows:

Theorem 1.1. [Non-positively curved case] Let $(M, g)$ be an $n$-dimensional CartanHadamard manifold (simply connected, complete Riemannian manifold with non-positive sectional curvature).

(i) [Sharpness] The Heisenberg-Pauli-Weyl principle $(\mathbf{H P W})_{x_{0}}$ holds for every $x_{0} \in$ $M$; moreover, $\frac{n^{2}}{4}$ is sharp, i.e.,

$$
\frac{n^{2}}{4}=\inf _{u \in C_{0}^{\infty}(M) \backslash\{0\}} \frac{\left(\int_{M}\left|\nabla_{g} u\right|^{2} \mathrm{~d} V_{g}\right)\left(\int_{M} d_{x_{0}}^{2} u^{2} \mathrm{~d} V_{g}\right)}{\left(\int_{M} u^{2} \mathrm{~d} V_{g}\right)^{2}} .
$$

(ii) [Extremals] The following statements are equivalent:

(a) $\frac{n^{2}}{4}$ is achieved by a positive extremal in $(\mathbf{H P W})_{x_{0}}$ for some $x_{0} \in M$;

(b) $\frac{n^{2}}{4}$ is achieved by a positive extremal in $(\mathbf{H P W})_{x_{0}}$ for every $x_{0} \in M$;

(c) $(M, g)$ is isometric to $\mathbb{R}^{n}$.

Some remarks are in order concerning Theorem 1.1.

Remark 1.1. (a) $(\mathbf{H P W})_{x_{0}}$ is a consequence of a quantitative/weighted HeisenbergPauli-Weyl principle stated below in Theorem 3.1. Note that similar weighted HeisenbergPauli-Weyl type principles have been investigated on Riemannian manifolds diffeomorphic to $\mathbb{R}^{n}$ (thus, in particular, on Cartan-Hadamard manifolds). Indeed, by using an operator theoretic approach, Erb [18, Theorem 2.54] stated weighted Heisenberg-Pauli-Weyl principles where the weights are in terms of volume distortion coefficients involving information 
on the curvature of the manifold. By using Bishop-Gromov comparison arguments, Corollary 2.68 of Erb [18] can be seen as $(\mathbf{H P W})_{x_{0}}$. Note however that the sharpness and the characterization of extremals in $(\mathbf{H P W})_{x_{0}}$ are not explicitly investigated in [18].

(b) One could expect finer results for $(\mathbf{H P W})_{x_{0}}$ whenever the Riemannian manifold is the model hyperbolic space. Andersen $[2,3]$ proved that hyperbolic Gaussians are candidates for extremal functions in Heisenberg-Pauli-Weyl principles within the hyperbolic setting. Recently, Kombe and Özaydin [29, Theorem 4.2] claimed that the hyperbolic Gaussian function $u(x)=e^{-\alpha d(x)^{2}}$ (where $\alpha>0$ is a root of a highly nonlinear equation) is an extremal function in the Heisenberg-Pauli-Weyl principle $(\mathbf{H P W})_{0}$ on the hyperbolic space $\mathbb{H}^{n}$; hereafter, $d(x)=d_{\mathbb{H} n}(0, x)$ denotes the hyperbolic distance between 0 and $x$ in the Poincaré ball model. According to Theorem 1.1, the scenario described in [29] cannot occur; moreover, two further independent arguments are presented in $\S 3.2$ which confirm the fact that in the hyperbolic setting the expected Gaussian function $u(x)=e^{-\alpha d(x)^{2}}$ cannot be extremal in $(\mathbf{H P W})_{0}$ for any $\alpha>0$. More precisely, the hyperbolic Gaussians are extremals for a quantitative Heisenberg-Pauli-Weyl principle rather than for $(\mathbf{H P W})_{0}$, as we shall explain in the sequel, see (3.13).

(c) Being within the context of Cartan-Hadamard manifolds, the sharpness of Sobolevtype inequalities usually require the validity of the longstanding Cartan-Hadamard conjecture, i.e., the sharp isoperimetric inequality (which is valid in 2, 3 and 4-dimensional Cartan-Hadamard manifolds), see e.g. Hebey [24, Section 8.2]. We notice that such a hypothesis is not needed in Theorem 1.1.

In the non-negatively curved case the situation is even more rigid than in Theorem 1.1:

Theorem 1.2. [Non-negatively curved case] Let $(M, g)$ be a complete, $n$-dimensional Riemannian manifold with non-negative Ricci curvature. The following statements are equivalent:

(a) $(\mathbf{H P W})_{x_{0}}$ holds for some $x_{0} \in M$;

(b) $(\mathbf{H P W})_{x_{0}}$ holds for every $x_{0} \in M$;

(c) $(M, g)$ is isometric to $\mathbb{R}^{n}$.

Remark 1.2. Theorem 1.2 can be included into the best constant program initiated by Aubin [4], and studied by Ledoux [30], Cheeger and Colding [12], Druet, Hebey and Vaugon [16], do Carmo and Xia [15], Minerbe [32], Li and Wang [31], etc. Indeed, in the aforementioned papers, the authors established that complete Riemannian manifolds with non-negative Ricci curvature supporting some Sobolev-type inequalities should be close to Euclidean spaces whenever the constant is sufficiently close to the sharp Euclidean Sobolev constant. The reader may consult Hebey [24] for a thoroughgoing presentation of this subject.

In the sequel, we shall present some closely related results to the sharp HeisenbergPauli-Weyl principle on Riemannian manifolds which are of independent interests.

Let $p, q \in \mathbb{R}$ and $n \in \mathbb{N}$ be such that

$$
0<q<2<p \text { and } 2<n<\frac{2(p-q)}{p-2} \text {. }
$$

For a fixed $x_{0} \in M$, we consider the Caffarelli-Kohn-Nirenberg interpolation inequality on $(M, g)$ : for all $u \in C_{0}^{\infty}(M)$,

$$
\left(\int_{M}\left|\nabla_{g} u\right|^{2} \mathrm{~d} V_{g}\right)\left(\int_{M} \frac{|u|^{2 p-2}}{d_{x_{0}}^{2 q-2}} \mathrm{~d} V_{g}\right) \geq \frac{(n-q)^{2}}{p^{2}}\left(\int_{M} \frac{|u|^{p}}{d_{x_{0}}^{q}} \mathrm{~d} V_{g}\right)^{2} .
$$


An endpoint of $(\mathbf{C K N})_{x_{0}}$ is precisely the Heisenberg-Pauli-Weyl principle $(\mathbf{H P W})_{x_{0}}$ whenever $p \rightarrow 2$ and $q \rightarrow 0$. As a part of the best constant program, Xia [39] proved that if $(M, g)$ is a complete, $n$-dimensional Riemannian manifold with non-negative Ricci curvature, then $(M, g)$ supports $(\mathbf{C K N})_{x_{0}}$ for some $x_{0} \in M$ if and only if $(M, g)$ is isometric to $\mathbb{R}^{n}$. In the Euclidean setting, Xia [39] also proved the sharpness of $\frac{(n-q)^{2}}{p^{2}}$ in $(\mathbf{C K N})_{x_{0}}$ and the existence of a class of extremals

$$
u_{\lambda}(x)=\left(\lambda+\left|x-x_{0}\right|^{2-q}\right)^{\frac{1}{2-p}}, \lambda>0 .
$$

The reader may also consult Kristály and Ohta [27] for a study of Caffarelli-KohnNirenberg inequalities on 'positively curved' metric measure spaces.

The non-positively curved counterpart of Xia's result, similar to Theorem 1.1, can be stated as follows:

Theorem 1.3. Let $p, q \in \mathbb{R}$ and $n \in \mathbb{N}$ be such that (1.2) holds and let $(M, g)$ be an n-dimensional Cartan-Hadamard manifold.

(i) [Sharpness] The Caffarelli-Kohn-Nirenberg interpolation inequality $(\mathbf{C K N})_{x_{0}}$ holds for every $x_{0} \in M$ and the constant $\frac{(n-q)^{2}}{p^{2}}$ is sharp, i.e.,

$$
\frac{(n-q)^{2}}{p^{2}}=\inf _{u \in C_{0}^{\infty}(M) \backslash\{0\}} \frac{\left(\int_{M}\left|\nabla_{g} u\right|^{2} \mathrm{~d} V_{g}\right)\left(\int_{M} \frac{|u|^{2 p-2}}{d_{x_{0}}^{2 q-2}} \mathrm{~d} V_{g}\right)}{\left(\int_{M} \frac{|u|^{p}}{d_{x_{0}}^{q}} \mathrm{~d} V_{g}\right)^{2}} .
$$

(ii) [Extremals] The following statements are equivalent:

(a) $\frac{(n-q)^{2}}{p^{2}}$ is achieved by a positive extremal in $(\mathbf{C K N})_{x_{0}}$ for some $x_{0} \in M$;

(b) $\frac{(n-q)^{2}}{p^{2}}$ is achieved by a positive extremal in $(\mathbf{C K N})_{x_{0}}$ for every $x_{0} \in M$;

(c) $(M, g)$ is isometric to $\mathbb{R}^{n}$.

The other endpoint of $(\mathbf{C K N})_{x_{0}}$, whenever $p \rightarrow 2$ and $q \rightarrow 2$, is the famous HardyPoincaré inequality on $(M, g)$ : for all $u \in C_{0}^{\infty}(M)$,

$$
\int_{M}\left|\nabla_{g} u\right|^{2} \mathrm{~d} V_{g} \geq \frac{(n-2)^{2}}{4} \int_{M} \frac{u^{2}}{d_{x_{0}}^{2}} \mathrm{~d} V_{g}
$$

In the Euclidean setting it is well known that $\frac{(n-2)^{2}}{4}$ is sharp, but there are no extremal functions. The lack of extremals motivated various improvements of the Hardy-Poincaré inequality; see e.g. Adimurthi, Chaudhuri and Ramaswamy [1], Barbatis, Filippas and Tertikas [5], Brezis and Vázquez [8], Filippas and Tertikas [20], Ghoussoub and Moradifam [22, 23], Wang and Willem [36], etc.

In the last few years, the Hardy-Poincaré inequality has been also studied on complete, non-compact Riemannian manifolds, where the influence of geometry played a key role; see e.g. Berchio, D’Ambrosio, Ganguly and Grillo [6], Berchio, Ganguly and Grillo [7], Carron [11], D'Ambrosio and Dipierro [14], Kombe and Özaydin [28, 29], Yang, Su and Kong [40], and references therein.

Our aim is to provide a new type of improved Hardy-Poincaré inequality which shows that more curvature implies more powerful improvements:

Theorem 1.4. [Improved Hardy-Poincaré inequality via curvature] Let $(M, g)$ be an $n$-dimensional Cartan-Hadamard manifold such that the sectional curvature is bounded from above by $c \leq 0$. Then for every $x_{0} \in M$ and $u \in C_{0}^{\infty}(M)$, we have

$$
\int_{M}\left|\nabla_{g} u\right|^{2} \mathrm{~d} V_{g} \geq \frac{(n-2)^{2}}{4} \int_{M} \frac{u^{2}}{d_{x_{0}}^{2}} \mathrm{~d} V_{g}+\frac{3|c|(n-1)(n-2)}{2} \int_{M} \frac{u^{2}}{\pi^{2}+|c| d_{x_{0}}^{2}} \mathrm{~d} V_{g} .
$$


In addition, the constant $\frac{(n-2)^{2}}{4}$ is sharp (independently by the second term on the RHS).

Remark 1.3. It seems similar rigidity results for the Hardy-Poincaré inequalities as in the Theorem 1.2 cannot be established on non-negatively curved spaces. In the proof of Theorem 1.2 the existence of extremals in the Euclidean case is crucial which fails in the case of Hardy-Poincaré inequalities.

Plan of the paper. In Section 2 we first recall the notions and results from Riemannian geometry which are used throughout the proofs. In Section 3 we first deal with the generic Heisenberg-Pauli-Weyl principle by proving Theorems 1.1\&1.2, and then we consider this principle on hyperbolic spaces (w.r.t. the paper [29]). In Section 4 we study related inequalities to the Heisenberg-Pauli-Weyl principle on Cartan-Hadamard manifolds (i.e., Caffarelli-Kohn-Nirenberg interpolation inequality and Hardy-Poincaré inequality).

\section{Preliminaries}

Let $(M, g)$ be an $n$-dimensional complete Riemannian manifold, and $d: M \times M \rightarrow$ $[0, \infty)$ be the metric function associated to the Riemannian metric $g$. Let $B(x, \rho)=\{y \in$ $M: d(x, y)<\rho\}$ be the open metric ball with center $x \in M$ and radius $\rho>0$. If $\mathrm{d} V_{g}$ is the canonical volume element on $(M, g)$, the volume of a bounded open set $S \subset M$ is

$$
\operatorname{Vol}_{g}(S)=\int_{S} \mathrm{~d} V_{g}=\operatorname{Haus}_{d}(S),
$$

where $\operatorname{Haus}_{d}(S)$ is the Hausdorff measure of $S$ with respect to the metric function $d$. In general, one has for every $x \in M$ that

$$
\lim _{\rho \rightarrow 0^{+}} \frac{\operatorname{Vol}_{g}(B(x, \rho))}{\omega_{n} \rho^{n}}=1
$$

where $\omega_{n}$ is the volume of the standard $n$-dimensional Euclidean unit ball.

Let $u: M \rightarrow \mathbb{R}$ be of class $C^{1}$. If $\left(x^{i}\right)$ is the local coordinate system on a coordinate neighborhood of $x \in M$, and the local components of the differential of $u$ are denoted $u_{i}=\frac{\partial u}{\partial x_{i}}$, then the local components of the gradient $\nabla_{g} u$ are $u^{i}=g^{i j} u_{j}$. Here, $g^{i j}$ are the local components of $g^{-1}=\left(g_{i j}\right)^{-1}$.

The Laplace-Beltrami operator is given by $\Delta_{g} u=\operatorname{div}\left(\nabla_{g} u\right)$ whose expression in a local chart of associated coordinates $\left(x^{i}\right)$ is

$$
\Delta_{g} u=g^{i j}\left(\frac{\partial^{2} u}{\partial x_{i} \partial x_{j}}-\Gamma_{i j}^{k} \frac{\partial u}{\partial x_{k}}\right)
$$

where $\Gamma_{i j}^{k}$ are the coefficients of the Levi-Civita connection.

If $u, v: M \rightarrow \mathbb{R}$ are of class $C^{2}$, one has the following integration by parts formula

$$
\int_{M} v \Delta_{g} u \mathrm{~d} V_{g}=-\int_{M}\left\langle\nabla_{g} v, \nabla_{g} u\right\rangle \mathrm{d} V_{g},
$$

where $\langle\cdot, \cdot\rangle$ denotes the scalar product associated with the Riemannian metric $g$ for 1-forms. For simplicity, we shall use the notation $|\alpha|=\sqrt{\langle\alpha, \alpha\rangle}$ for any 1 -form.

A Riemannian manifold $(M, g)$ is called Cartan-Hadamard if it is complete, simply connected and with non-positive sectional curvature.

For every $c \leq 0$ we consider the function $\mathbf{c t}_{c}:(0, \infty) \rightarrow \mathbb{R}$ defined by

$$
\operatorname{ct}_{c}(\rho)= \begin{cases}\frac{1}{\rho} & \text { if } c=0, \\ \sqrt{|c|} \operatorname{coth}(\sqrt{|c|} \rho) & \text { if } c<0 .\end{cases}
$$


For further use, let $\mathbf{D}_{c}:[0, \infty) \rightarrow \mathbb{R}$ defined by

$$
\mathbf{D}_{c}(\rho)= \begin{cases}0 & \text { if } \rho=0 \\ \rho \mathbf{c t}_{c}(\rho)-1 & \text { if } \rho>0 .\end{cases}
$$

It is clear that $\mathbf{D}_{c} \geq 0$.

Hereafter, $d_{x_{0}}(x)=d\left(x_{0}, x\right)$ denotes the distance function from a given point $x_{0} \in M$.

Theorem 2.1. [Laplacian comparison; see [38, Theorem 5.1]] Let $(M, g)$ be an $n-d i$ mensional Cartan-Hadamard manifold such that the sectional curvature is bounded from above by $c \leq 0$, and let $x_{0} \in M$ be fixed. Then we have (in distributional sense) that

$$
\Delta_{g} d_{x_{0}} \geq(n-1) \mathbf{c t}_{c}\left(d_{x_{0}}\right) .
$$

In the proof of our results Bishop-Gromov-type volume comparison principles play a crucial role. Here we adapt from the Finsler version the following form (see Shen [35], $\mathrm{Wu}$ and Xin [38, Theorems $6.1 \& 6.3]$ and Zhao and Shen [41]):

Theorem 2.2. [Volume comparison] Let $(M, g)$ be a complete, $n$-dimensional Riemannian manifold. Then the following statements hold.

(a) If $(M, g)$ is a Cartan-Hadamard manifolds, the function $\rho \mapsto \frac{\operatorname{Vol}_{g}(B(x, \rho))}{\rho^{n}}$ is nondecreasing, $\rho>0$. In particular, from (2.1) we have

$$
\operatorname{Vol}_{g}(B(x, \rho)) \geq \omega_{n} \rho^{n} \text { for all } x \in M \text { and } \rho>0 .
$$

If equality holds in (2.2), then the sectional curvature is identically zero.

(b) If $(M, g)$ has non-negative Ricci curvature, the function $\rho \mapsto \frac{\operatorname{Vol}_{g}(B(x, \rho))}{\rho^{n}}$ is nonincreasing, $\rho>0$. In particular, from (2.1) we have

$$
\operatorname{Vol}_{g}(B(x, \rho)) \leq \omega_{n} \rho^{n} \text { for all } x \in M \text { and } \rho>0 .
$$

If equality holds in (2.3), then the sectional curvature is identically zero.

\section{Heisenberg-Pauli-Weyl PRinciple on Riemannian manifolds}

3.1. Non-positively curved case: proof of Theorem 1.1. First, we present a quantitative version of the Heisenberg-Pauli-Weyl principle.

Theorem 3.1. [Quantitative Heisenberg-Pauli-Weyl principle] Let $(M, g)$ be an $n$-dimensional Cartan-Hadamard manifold such that the sectional curvature is bounded from above by $c \leq 0$. Then for all $x_{0} \in M$ and $u \in C_{0}^{\infty}(M)$, we have

$$
\left(\int_{M}\left|\nabla_{g} u\right|^{2} \mathrm{~d} V_{g}\right)\left(\int_{M} d_{x_{0}}^{2} u^{2} \mathrm{~d} V_{g}\right) \geq \frac{n^{2}}{4}\left(\int_{M}\left(1+\frac{n-1}{n} \mathbf{D}_{c}\left(d_{x_{0}}\right)\right) u^{2} \mathrm{~d} V_{g}\right)^{2} .
$$

Proof. Let $x_{0} \in M$ and $u \in C_{0}^{\infty}(M)$ be fixed arbitrarily. According to Theorem 2.1, one has

$$
\begin{aligned}
\int_{M} \Delta_{g}\left(d_{x_{0}}^{2}\right) u^{2} \mathrm{~d} V_{g} & =2 \int_{M}\left(1+d_{x_{0}} \Delta_{g} d_{x_{0}}\right) u^{2} \mathrm{~d} V_{g} \\
& \geq 2 \int_{M}\left(1+(n-1) d_{x_{0}} \mathbf{c t}_{c}\left(d_{x_{0}}\right)\right) u^{2} \mathrm{~d} V_{g} \\
& =2 n \int_{M}\left(1+\frac{n-1}{n} \mathbf{D}_{c}\left(d_{x_{0}}\right)\right) u^{2} \mathrm{~d} V_{g}
\end{aligned}
$$


An integration by parts yields

$$
\begin{aligned}
\int_{M} \Delta_{g}\left(d_{x_{0}}^{2}\right) u^{2} \mathrm{~d} V_{g} & =-\int_{M}\left\langle\nabla_{g}\left(u^{2}\right), \nabla_{g}\left(d_{x_{0}}^{2}\right)\right\rangle \mathrm{d} V_{g} \\
& =-4 \int_{M} u d_{x_{0}}\left\langle\nabla_{g} u, \nabla_{g} d_{x_{0}}\right\rangle \mathrm{d} V_{g} .
\end{aligned}
$$

By the eikonal equation $\left|\nabla_{g} d_{x_{0}}\right|=1$ a.e. on $M$, one has that $\left|\left\langle\nabla_{g} u, \nabla_{g} d_{x_{0}}\right\rangle\right| \leq\left|\nabla_{g} u\right|$. Thus, by Schwartz inequality one gets

$$
\left(\int_{M} u d_{x_{0}}\left\langle\nabla_{g} u, \nabla_{g} d_{x_{0}}\right\rangle \mathrm{d} V_{g}\right)^{2} \leq\left(\int_{M} d_{x_{0}}^{2} u^{2} \mathrm{~d} V_{g}\right)\left(\int_{M}\left|\nabla_{g} u\right|^{2} \mathrm{~d} V_{g}\right) .
$$

The latter relation coupled with (3.1) yields the quantitative Heisenberg-Pauli-Weyl principle, which concludes the proof.

Proof of Theorem 1.1. (i) Let $x_{0} \in M$ be fixed. Since $\mathbf{D}_{c} \geq 0$, due to Theorem 3.1, the Heisenberg-Pauli-Weyl principle $(\mathbf{H P W})_{x_{0}}$ holds.

We shall prove that the constant $\frac{n^{2}}{4}$ is optimal in $(\mathbf{H P W})_{x_{0}}$, following Aubin's argument [4]; see also Hebey [24]. Let

$$
\mathrm{C}_{\mathrm{HPW}}=\inf _{u \in C_{0}^{\infty}(M) \backslash\{0\}} \frac{\left(\int_{M}\left|\nabla_{g} u\right|^{2} \mathrm{~d} V_{g}\right)\left(\int_{M} d_{x_{0}}^{2} u^{2} \mathrm{~d} V_{g}\right)}{\left(\int_{M} u^{2} \mathrm{~d} V_{g}\right)^{2}} .
$$

Since $(\mathbf{H P W})_{x_{0}}$ holds, then $\mathrm{C}_{\mathrm{HPW}} \geq \frac{n^{2}}{4}$. Assume that $\mathrm{C}_{\mathrm{HPW}}>\frac{n^{2}}{4}$. By (3.2), one has

$$
\left(\int_{M}\left|\nabla_{g} u\right|^{2} \mathrm{~d} V_{g}\right)\left(\int_{M} d_{x_{0}}^{2} u^{2} \mathrm{~d} V_{g}\right) \geq \mathrm{C}_{\text {HPW }}\left(\int_{M} u^{2} \mathrm{~d} V_{g}\right)^{2}, \forall u \in C_{0}^{\infty}(M) .
$$

For every $\varepsilon>0$, there exists a local chart $(\Omega, \phi)$ of $M$ at the point $x_{0}$ and a number $\delta>0$ such that $\phi(\Omega)=B_{e}(0, \delta)$ and the components $g_{i j}$ of the metric $g$ satisfy

$$
(1-\varepsilon) \delta_{i j} \leq g_{i j} \leq(1+\varepsilon) \delta_{i j}
$$

in the sense of bilinear forms. Here, $B_{e}(0, \delta)$ is the $n$-dimensional Euclidean ball of center 0 and radius $\delta>0$.

According to (3.3) and to the two-sided metric estimate (3.4), for $\varepsilon>0$ small enough, there exists $\tilde{\delta}>0$ and $C_{\mathrm{HPW}}^{\prime}>\frac{n^{2}}{4}$ such that for every $\delta \in(0, \tilde{\delta})$ and $w \in C_{0}^{\infty}\left(B_{e}(0, \delta)\right)$,

$$
\left(\int_{B_{e}(0, \delta)}|\nabla w|^{2} \mathrm{~d} x\right)\left(\int_{B_{e}(0, \delta)}|x|^{2} w^{2} \mathrm{~d} x\right) \geq \mathrm{C}_{\mathrm{HPW}}^{\prime}\left(\int_{B_{e}(0, \delta)} w^{2} \mathrm{~d} x\right)^{2} .
$$

Let $u \in C_{0}^{\infty}\left(\mathbb{R}^{n}\right)$ be fixed arbitrarily and set $w_{\lambda}(x)=u(\lambda x), \lambda>0$. It is clear that $w_{\lambda} \in C_{0}^{\infty}\left(B_{e}(0, \delta)\right)$ for enough large $\lambda>0$. Inserting $w_{\lambda}$ into (3.5), and having the scaling properties

$$
\int_{B_{e}(0, \delta)}\left|\nabla w_{\lambda}\right|^{2} \mathrm{~d} x=\lambda^{2-n} \int_{\mathbb{R}^{n}}|\nabla u|^{2} \mathrm{~d} x, \int_{B_{e}(0, \delta)}|x|^{2} w_{\lambda}^{2} \mathrm{~d} x=\lambda^{-2-n} \int_{\mathbb{R}^{n}}|x|^{2} u^{2} \mathrm{~d} x,
$$

and

it follows that

$$
\int_{B_{e}(0, \delta)} w_{\lambda}^{2} \mathrm{~d} x=\lambda^{-n} \int_{\mathbb{R}^{n}} u^{2} \mathrm{~d} x
$$

$$
\left(\int_{\mathbb{R}^{n}}|\nabla u|^{2} \mathrm{~d} x\right)\left(\int_{\mathbb{R}^{n}}|x|^{2} u^{2} \mathrm{~d} x\right) \geq \mathrm{C}_{\mathrm{HPW}}^{\prime}\left(\int_{\mathbb{R}^{n}} u^{2} \mathrm{~d} x\right)^{2} .
$$


In particular, in the latter relation we may substitute the Gaussian function $u(x)=e^{-|x|^{2}}$, obtaining that $\frac{n^{2}}{4} \geq \mathrm{C}_{\mathrm{HPW}}^{\prime}$, a contradiction. Consequently, $\mathrm{C}_{\mathrm{HPW}}=\frac{n^{2}}{4}$.

(ii) First, if $(M, g)$ is isometric to $\mathbb{R}^{n}$, the sharp Heisenberg-Pauli-Weyl principle $(\mathbf{H P W})_{x_{0}}$ can be equivalently transformed into (1.1) for which the Gaussian functions $u_{\lambda}(x)=$ $e^{-\lambda|x|^{2}}, \lambda>0$, are extremal functions. Thus, the implications (c) $\Rightarrow(\mathrm{b}) \Rightarrow(\mathrm{a})$ hold true.

We now prove $(\mathrm{a}) \Rightarrow(\mathrm{c})$. Let $u_{0}>0$ be an extremal function in the sharp HeisenbergPauli-Weyl principle $(\mathbf{H P W})_{x_{0}}$ for some $x_{0} \in M$. In particular, in the estimates in Theorem 3.1 we should have equalities; thus, by (3.1) one has $\mathbf{D}_{c} \equiv 0$ (i.e., we necessarily have $c=0$, so the sectional curvature of $(M, g)$ cannot be bounded above by a fixed negative number), and

$$
\Delta_{g}\left(d_{x_{0}}^{2}\right)=2 n
$$

Let us fix $\rho>0$ arbitrarily. Note that the unit outward pointing normal vector to the sphere $S\left(x_{0}, \rho\right)=\partial B\left(x_{0}, \rho\right)=\left\{x \in M: d\left(x_{0}, x\right)=\rho\right\}$ is given by $\mathbf{n}=\nabla_{g} d_{x_{0}}$. Let us denote by $\mathrm{d} \varsigma_{g}$ the volume form on $S\left(x_{0}, \rho\right)$ induced from $\mathrm{d} V_{g}$. By applying Stokes' formula and the fact that $\langle\mathbf{n}, \mathbf{n}\rangle=1$ we have

$$
\begin{aligned}
2 n \operatorname{Vol}_{g}\left(B\left(x_{0}, \rho\right)\right) & =\int_{B\left(x_{0}, \rho\right)} \Delta_{g}\left(d_{x_{0}}^{2}\right) \mathrm{d} V_{g}=\int_{B\left(x_{0}, \rho\right)} \operatorname{div}\left(\nabla_{g}\left(d_{x_{0}}^{2}\right)\right) \mathrm{d} V_{g} \\
& =\int_{S\left(x_{0}, \rho\right)}\left\langle\mathbf{n}, \nabla_{g}\left(d_{x_{0}}^{2}\right)\right\rangle \mathrm{d} \varsigma_{g}=2 \int_{S\left(x_{0}, \rho\right)} d_{x_{0}}\left\langle\mathbf{n}, \nabla_{g} d_{x_{0}}\right\rangle \mathrm{d} \varsigma_{g} \\
& =2 \rho \int_{S\left(x_{0}, \rho\right)}\langle\mathbf{n}, \mathbf{n}\rangle \mathrm{d} \varsigma_{g}=2 \rho \int_{S\left(x_{0}, \rho\right)} \mathrm{d} \varsigma_{g} \\
& =2 \rho \mathrm{A}_{g}\left(S\left(x_{0}, \rho\right)\right),
\end{aligned}
$$

where

$$
\mathrm{A}_{g}\left(S\left(x_{0}, \rho\right)\right)=\lim _{\varepsilon \rightarrow 0^{+}} \frac{\operatorname{Vol}_{g}\left(B\left(x_{0}, \rho+\varepsilon\right)\right)-\operatorname{Vol}_{g}\left(B\left(x_{0}, \rho\right)\right)}{\varepsilon}:=\frac{\mathrm{d}}{\mathrm{d} \rho} \operatorname{Vol}_{g}\left(B\left(x_{0}, \rho\right)\right)
$$

is the surface area of $S\left(x_{0}, \rho\right)$. Thus, the above relations imply that

$$
\frac{\frac{\mathrm{d}}{\mathrm{d} \rho} \operatorname{Vol}_{g}\left(B\left(x_{0}, \rho\right)\right)}{\operatorname{Vol}_{g}\left(B\left(x_{0}, \rho\right)\right)}=\frac{n}{\rho} .
$$

By integrating this expression and due to relation (2.1), we conclude that

$$
\operatorname{Vol}_{g}\left(B\left(x_{0}, \rho\right)\right)=\omega_{n} \rho^{n} \text { for all } \rho>0 .
$$

Let $x \in M$ and $\rho>0$ be arbitrarily fixed. Since $(M, g)$ is of Cartan-Hadamard type, by the volume comparison (see Theorem 2.2(a)), the function $r \mapsto \frac{\operatorname{Vol}_{g}(B(x, r))}{r^{n}}$ is nondecreasing on $(0, \infty)$. Therefore, one has

$$
\begin{aligned}
\omega_{n} & \leq \frac{\operatorname{Vol}_{g}(B(x, \rho))}{\rho^{n}} \\
& \leq \limsup _{r \rightarrow \infty} \frac{\operatorname{Vol}_{g}(B(x, r))}{r^{n}} \quad \text { (monotonicity) } \\
& \leq \limsup _{r \rightarrow \infty} \frac{\operatorname{Vol}_{g}\left(B\left(x_{0}, r+d\left(x_{0}, x\right)\right)\right)}{r^{n}} \quad\left(B(x, r) \subset B\left(x_{0}, r+d\left(x_{0}, x\right)\right)\right) \\
& =\limsup _{r \rightarrow \infty}\left(\frac{\operatorname{Vol}_{g}\left(B\left(x_{0}, r+d\left(x_{0}, x\right)\right)\right)}{\left(r+d\left(x_{0}, x\right)\right)^{n}} \cdot \frac{\left(r+d\left(x_{0}, x\right)\right)^{n}}{r^{n}}\right) \\
& =\omega_{n} .
\end{aligned}
$$

Consequently,

$$
\operatorname{Vol}_{g}(B(x, \rho))=\omega_{n} \rho^{n} \text { for all } x \in M \text { and } \rho>0 \text {. }
$$


Now, the equality case in Theorem 2.2(a) implies that the sectional curvature is identically zero, which concludes the proof.

Remark 3.1. Implication $(\mathrm{a}) \Rightarrow(\mathrm{c})$ in Theorem 1.1 has also a geometric proof. Indeed, due to Jost [26, Lemma 2.1.5] and relation (3.6), it follows that we have equality in the $\mathrm{CAT}(0)$-inequality with reference point $x_{0} \in M$, i.e., for every geodesic segment $\gamma:[0,1] \rightarrow M$ and $s \in[0,1]$, we have

$$
d^{2}\left(x_{0}, \gamma(s)\right)=(1-s) d^{2}\left(x_{0}, \gamma(0)\right)+s d^{2}\left(x_{0}, \gamma(1)\right)-s(1-s) d^{2}(\gamma(0), \gamma(1)) .
$$

Now, Alexandrov's rigidity result implies that the geodesic triangle formed by the points $x_{0}, \gamma(0)$ and $\gamma(1)$ is flat, see e.g. Bridson and Haefliger [9]. Therefore, the conclusion that $(M, g)$ is isometric to the Euclidean space $\mathbb{R}^{n}$ follows in a standard manner; the author thanks J. Jost and A. Lytchak for pointing out this approach.

3.2. Sharp Heisenberg-Pauli-Weyl principle on hyperbolic spaces. For the hyperbolic space we use the Poincaré ball model $\mathbb{H}^{n}=\left\{x \in \mathbb{R}^{n}:|x|<1\right\}$ endowed with the Riemannian metric

$$
g_{\text {hyp }}(x)=\left(g_{i j}(x)\right)_{i, j=1, \ldots, n}=p(x)^{2} \delta_{i j},
$$

where $p(x)=\frac{2}{1-|x|^{2}}$. It is well known that $\left(\mathbb{H}^{n}, g_{\mathrm{hyp}}\right)$ is a Cartan-Hadamard manifold with constant sectional curvature -1 . The volume form is

$$
\mathrm{d} V_{\mathbb{H}^{n}}(x)=p(x)^{n} \mathrm{~d} x,
$$

while the hyperbolic gradient and Laplace-Beltrami operator are given by

$$
\nabla_{\mathbb{H}^{n}} u=\frac{\nabla u}{p^{2}} \text { and } \Delta_{\mathbb{H}^{n}} u=p^{-n} \operatorname{div}\left(p^{n-2} \nabla u\right),
$$

where $\nabla$ denotes the Euclidean gradient in $\mathbb{R}^{n}$. The hyperbolic distance between the origin and $x \in \mathbb{H}^{n}$ is given by

$$
d_{\mathbb{H}^{n}}(0, x)=\ln \left(\frac{1+|x|}{1-|x|}\right) .
$$

Recently, Kombe and Özaydin [29] stated a Heisenberg-Pauli-Weyl principle on $\left(\mathbb{H}^{n}, g_{\text {hyp }}\right)$. For completeness, we recall the statement of Theorem 4.2 from [29]:

"Let $u \in C_{0}^{\infty}\left(\mathbb{H}^{n}\right), d=d(x)=d_{\mathbb{H}^{n}}(0, x)$ and $n>2$. Then

$$
\left(\int_{\mathbb{H}^{n}}\left|\nabla_{\mathbb{H}^{n}} u\right|^{2} \mathrm{~d} V_{\mathbb{H}^{n}}\right)\left(\int_{\mathbb{H}^{n}} d^{2} u^{2} \mathrm{~d} V_{\mathbb{H}^{n}}\right) \geq \frac{n^{2}}{4}\left(\int_{\mathbb{H}^{n}} u^{2} \mathrm{~d} V_{\mathbb{H}^{n}}\right)^{2} .
$$

Moreover, equality holds in (3.10) if $u(x)=A e^{-\alpha d^{2}}$, where $A \in \mathbb{R}$, and

$$
\alpha=\frac{n-1}{n-2}\left(n-1+2 \pi \frac{C_{n-2}}{C_{n}}\right)
$$

with $C_{n}=\int_{\mathbb{H}^{n}} e^{-\alpha d^{2}} \mathrm{~d} V_{\mathbb{H}^{n}}, \alpha>0 . "$

Relation (3.10) holds true, see also Theorem 1.1. However, the statement concerning the equality in (3.10) cannot happen, which has the following three independent proofs:

Argument 1 (based on the non-solvability of (3.11)) . Let $C_{n}=C_{n}(\alpha)=\int_{\mathbb{H}^{n}} e^{-\alpha d^{2}} \mathrm{~d} V_{\mathbb{H}^{n}}$ be as above. We claim that the non-linear equation (3.11) cannot be solved generically in 
$\alpha>0$. For simplicity, we consider only the case $n=4$; then equation (3.11) reduces to $\alpha=w(\alpha)$, where

$$
w(\alpha):=\frac{3}{2}\left(3+2 \pi \frac{\int_{\mathbb{H}^{2}} e^{-\alpha d^{2}} \mathrm{~d} V_{\mathbb{H}^{2}}}{\int_{\mathbb{H}^{4}} e^{-\alpha d^{2}} \mathrm{~d} V_{\mathbb{H}^{4}}}\right) .
$$

Since $w \geq \frac{9}{2}$, the values for $\alpha$ should belong to $\left[\frac{9}{2}, \infty\right)$ in order to solve $\alpha=w(\alpha)$.

We claim that

$$
w(\alpha) \geq 2 \alpha+1 \text { for every } \alpha \in[4, \infty),
$$

which will clearly imply the non-solvability of $\alpha=w(\alpha)$.

By (3.9), a change of variables shows that

$$
w(\alpha)=\frac{9}{2}+\frac{3 \int_{0}^{\infty} e^{-\alpha t^{2}} \sinh (t) \mathrm{d} t}{\int_{0}^{\infty} e^{-\alpha t^{2}} \sinh ^{3}(t) \mathrm{d} t}=\frac{9}{2}+\frac{12 \operatorname{erf}\left(\frac{1}{2 \sqrt{\alpha}}\right)}{e^{\frac{2}{\alpha}} \operatorname{erf}\left(\frac{3}{2 \sqrt{\alpha}}\right)-3 \operatorname{erf}\left(\frac{1}{2 \sqrt{\alpha}}\right)},
$$

where $\operatorname{erf}(s)=\frac{2}{\sqrt{\pi}} \int_{0}^{s} e^{-t^{2}} \mathrm{~d} t$ is the Gauss error function. Therefore, the claim (3.12) is equivalent to the inequality

$$
3 \frac{4 \alpha+1}{4 \alpha-7} e^{-\frac{2}{\alpha}} \operatorname{erf}\left(\frac{1}{2 \sqrt{\alpha}}\right) \geq \operatorname{erf}\left(\frac{3}{2 \sqrt{\alpha}}\right) .
$$

If $s=\frac{1}{2 \sqrt{\alpha}} \in\left(0, \frac{1}{4}\right]$, the latter inequality is equivalent to

$$
3 \frac{1+s^{2}}{1-7 s^{2}} e^{-8 s^{2}} \geq \frac{\operatorname{erf}(3 s)}{\operatorname{erf}(s)}, \quad s \in\left(0, \frac{1}{4}\right] .
$$

Simple estimates for the error and exponential functions give for every $s \in\left(0, \frac{1}{4}\right]$ that

$$
3 \frac{1+s^{2}}{1-7 s^{2}} \cdot e^{-8 s^{2}}-\frac{\operatorname{erf}(3 s)}{\operatorname{erf}(s)} \geq 3 \frac{\left(1+s^{2}\right)\left(1-4 s^{2}\right)^{2}}{1-7 s^{2}}-3 \frac{1-s^{2}}{1-\frac{1}{3} s^{2}} \geq 0,
$$

which concludes the proof of (3.12).

Argument 2 (based on Theorem 1.1). Following Kombe and Özaydin [29], let us assume that the hyperbolic Gaussian $u=e^{-\alpha d^{2}}>0$ is an extremal function in (3.10) for some $\alpha>0$. Due to Theorem 1.1 (ii), it follows that the hyperbolic space $\left(\mathbb{H}^{n}, g_{\text {hyp }}\right)$ is isometric to the standard Euclidean space $\mathbb{R}^{n}$, a contradiction.

Argument 3 (based on Theorem 3.1). Due to Theorem 3.1, for every $u \in C_{0}^{\infty}\left(\mathbb{H}^{n}\right)$ one has

$$
\left(\int_{\mathbb{H}^{n}}\left|\nabla_{\mathbb{H}^{n}} u\right|^{2} \mathrm{~d} V_{\mathbb{H}^{n}}\right)\left(\int_{\mathbb{H}^{n}} d^{2} u^{2} \mathrm{~d} V_{\mathbb{H}^{n}}\right) \geq \frac{n^{2}}{4}\left(\int_{\mathbb{H}^{n}}\left(1+\frac{n-1}{n} \mathbf{D}_{-1}(d)\right) u^{2} \mathrm{~d} V_{\mathbb{H}^{n}}\right)^{2} .
$$

Since $\mathbf{D}_{-1}(d) \geq 0$, if one expects to have equality in (3.10) for $u=e^{-\alpha d^{2}}$ for some $\alpha>0$, we necessarily have in (3.13) the relation $\mathbf{D}_{-1}(\rho)=0$ for every $\rho \geq 0$; this relation means that for every $\rho \geq 0$ we have

$$
0=\rho \mathbf{c t}_{-1}(\rho)-1=\rho \operatorname{coth}(\rho)-1,
$$


a contradiction. Moreover, in the inequality (3.13) the constant $\frac{n^{2}}{4}$ is sharp and an integration by parts easily shows (by using the exact form of the volume element (3.9)) that the equality holds for the hyperbolic Gaussian family of functions $u_{\alpha}=e^{-\alpha d^{2}}, \alpha>0$.

Summing up the above discussions, we conclude that:

The hyperbolic Gaussian functions $u_{\lambda}=e^{-\lambda d^{2}}, \lambda>0$, represent the family of extremals for the quantitative Heisenberg-Pauli-Weyl principle (3.13), but not for the 'pure' Heisenberg-Pauli-Weyl principle (3.10).

3.3. Non-negatively curved case: proof of Theorem 1.2. Implications $(\mathrm{c}) \Rightarrow(\mathrm{b}) \Rightarrow(\mathrm{a})$ trivially hold. The proof of the implication $(\mathrm{a}) \Rightarrow(\mathrm{c})$ is divided into four steps. Let $x_{0} \in M$ be fixed.

Step 1. If $(M, g)$ is isometric to $\mathbb{R}^{n}$, then $(\mathbf{H P W})_{x_{0}}$ can be transformed into the inequality (1.1) for which the standard class of Gaussian functions are extremals.

For later use, if we consider the function $T:(0, \infty) \rightarrow \mathbb{R}$ defined by

$$
T(\lambda)=\int_{\mathbb{R}^{n}} e^{-2 \lambda|x|^{2}} \mathrm{~d} x, \lambda>0,
$$

the equality for the family of extremals in (1.1) can be rewritten to the form

$$
-\lambda T^{\prime}(\lambda)=\frac{n}{2} T(\lambda), \lambda>0 .
$$

Moreover, by the layer cake representation and changing a variable, one has the following representations which are used later:

$$
T(\lambda)=4 \lambda \omega_{n} \int_{0}^{\infty} \rho^{n+1} e^{-2 \lambda \rho^{2}} \mathrm{~d} \rho=\frac{2}{(2 \lambda)^{\frac{n}{2}}} \omega_{n} \int_{0}^{\infty} t^{n+1} e^{-t^{2}} \mathrm{~d} t
$$

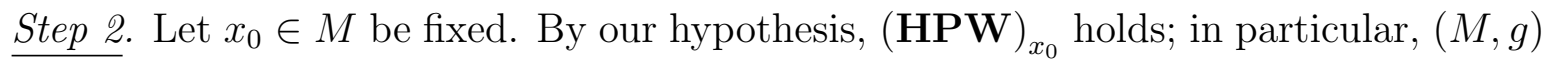
cannot be compact. We consider the class of functions

$$
\tilde{u}_{\lambda}(x)=e^{-\lambda d_{x_{0}}(x)^{2}}, \lambda>0 .
$$

Clearly, the function $\tilde{u}_{\lambda}$ can be approximated by elements from $C_{0}^{\infty}(M)$ for every $\lambda>0$. By inserting $\tilde{u}_{\lambda}$ into $(\mathbf{H P W})_{x_{0}}$, and using $\left|\nabla_{g} d_{x_{0}}\right|=1$ a.e. on $M$, we obtain that

$$
2 \lambda \int_{M} d_{x_{0}}^{2} e^{-2 \lambda d_{x_{0}}^{2}} \mathrm{~d} V_{g} \geq \frac{n}{2} \int_{M} e^{-2 \lambda d_{x_{0}}^{2}} \mathrm{~d} V_{g}, \lambda>0 .
$$

We introduce the function $\mathscr{T}:(0, \infty) \rightarrow \mathbb{R}$ defined by

$$
\mathscr{T}(\lambda)=\int_{M} e^{-2 \lambda d_{x_{0}}^{2}} \mathrm{~d} V_{g}, \lambda>0 .
$$

By the layer cake representation, $\mathscr{T}$ can be equivalently rewritten to

$$
\begin{aligned}
\mathscr{T}(\lambda) & =\int_{0}^{\infty} \operatorname{Vol}_{g}\left(\left\{x \in M: e^{-2 \lambda d_{x_{0}}^{2}}>t\right\}\right) \mathrm{d} t=\int_{0}^{1} \operatorname{Vol}_{g}\left(\left\{x \in M: e^{-2 \lambda d_{x_{0}}^{2}}>t\right\}\right) \mathrm{d} t \\
& =4 \lambda \int_{0}^{\infty} \operatorname{Vol}_{g}\left(B\left(x_{0}, \rho\right)\right) \rho e^{-2 \lambda \rho^{2}} \mathrm{~d} \rho .
\end{aligned}
$$

Since the Ricci curvature is non-negative, one account of (2.3), the function $\mathscr{T}$ is well defined and differentiable. Thus, relation (3.16) is equivalent to

$$
-\lambda \mathscr{T}^{\prime}(\lambda) \geq \frac{n}{2} \mathscr{T}(\lambda), \quad \lambda>0 .
$$


Step 3. We shall prove that

$$
\mathscr{T}(\lambda) \geq T(\lambda) \text { for all } \lambda>0
$$

By (3.14) and (3.17) it turns out that

$$
\frac{\mathscr{T}^{\prime}(\lambda)}{\mathscr{T}(\lambda)} \leq \frac{T^{\prime}(\lambda)}{T(\lambda)}, \lambda>0
$$

Integrating this inequality, it yields that the function $\lambda \mapsto \frac{\mathscr{T}(\lambda)}{T(\lambda)}$ is non-increasing; in particular, for every $\lambda>0$,

Now, we shall prove that

$$
\frac{\mathscr{T}(\lambda)}{T(\lambda)} \geq \liminf _{\lambda \rightarrow \infty} \frac{\mathscr{T}(\lambda)}{T(\lambda)}
$$

$$
\liminf _{\lambda \rightarrow \infty} \frac{\mathscr{T}(\lambda)}{T(\lambda)} \geq 1
$$

Due to relation (2.1), for every $\varepsilon>0$ one can find $\rho_{\varepsilon}>0$ such that

$$
\operatorname{Vol}_{g}\left(B\left(x_{0}, \rho\right)\right) \geq(1-\varepsilon) \omega_{n} \rho^{n} \text { for all } \rho \in\left[0, \rho_{\varepsilon}\right] \text {. }
$$

Consequently, one has

$$
\begin{aligned}
\mathscr{T}(\lambda) & =4 \lambda \int_{0}^{\infty} \operatorname{Vol}_{g}\left(B\left(x_{0}, \rho\right)\right) \rho e^{-2 \lambda \rho^{2}} \mathrm{~d} \rho \\
& \geq 4 \lambda(1-\varepsilon) \omega_{n} \int_{0}^{\rho_{\varepsilon}} \rho^{n+1} e^{-2 \lambda \rho^{2}} \mathrm{~d} \rho \\
& =\frac{2}{(2 \lambda)^{\frac{n}{2}}}(1-\varepsilon) \omega_{n} \int_{0}^{\sqrt{2 \lambda} \rho_{\varepsilon}} t^{n+1} e^{-t^{2}} \mathrm{~d} t . \quad(\sqrt{2 \lambda} \rho=t)
\end{aligned}
$$

Now, by (3.15), it yields that

$$
\liminf _{\lambda \rightarrow \infty} \frac{\mathscr{T}(\lambda)}{T(\lambda)} \geq 1-\varepsilon
$$

Since $\varepsilon>0$ is arbitrary, relation (3.20) holds, so (3.19). This ends the proof of the claim (3.18).

Step 4. Via (3.15) and the representation of $\mathcal{T}$, relation (3.18) is equivalent to

$$
\int_{0}^{\infty}\left(\operatorname{Vol}_{g}\left(B\left(x_{0}, \rho\right)\right)-\omega_{n} \rho^{n}\right) \rho e^{-2 \lambda \rho^{2}} \mathrm{~d} \rho \geq 0 \text { for all } \lambda>0 .
$$

Due to (2.3), we have

$$
\operatorname{Vol}_{g}\left(B\left(x_{0}, \rho\right)\right)=\omega_{n} \rho^{n} \text { for all } \rho>0 .
$$

Now, let $x \in M$ and $\rho>0$ be arbitrarily fixed. Note that by Theorem 2.2(b) the function $r \mapsto \frac{\operatorname{Vol}_{g}(B(x, r))}{r^{n}}$ is non-increasing on $(0, \infty)$. Therefore, we have

$$
\begin{array}{rlr}
\omega_{n} & \geq \frac{\operatorname{Vol}_{g}(B(x, \rho))}{\rho^{n}} & \text { (see }(2.3)) \\
& \geq \limsup _{r \rightarrow \infty} \frac{\operatorname{Vol}_{g}(B(x, r))}{r^{n}} & \text { (monotonicity) } \\
& \geq \limsup _{r \rightarrow \infty} \frac{\operatorname{Vol}_{g}\left(B\left(x_{0}, r-d\left(x_{0}, x\right)\right)\right)}{r^{n}} \quad\left(B(x, r) \supset B\left(x_{0}, r-d\left(x_{0}, x\right)\right)\right) \\
& =\limsup _{r \rightarrow \infty}\left(\frac{\operatorname{Vol}_{g}\left(B\left(x_{0}, r-d\left(x_{0}, x\right)\right)\right)}{\left(r-d\left(x_{0}, x\right)\right)^{n}} \cdot \frac{\left(r-d\left(x_{0}, x\right)\right)^{n}}{r^{n}}\right) \\
& =\omega_{n} . & \text { (see }(3.21))
\end{array}
$$


Consequently, one has

$$
\operatorname{Vol}_{g}(B(x, \rho))=\omega_{n} \rho^{n} \text { for all } x \in M, \rho \geq 0 .
$$

Thus, the equality case in Theorem 2.2(b) implies that the sectional curvature identically vanishes, which conludes the proof.

\section{Inequalities Related to the Heisenberg-Pauli-Weyl Principle on CARTAN-HADAMARD MANIFOLDS}

\subsection{Caffarelli-Kohn-Nirenberg interpolation inequality: proof of Theorem 1.3.} The proof is similar to Theorem 1.1.

(i) Let $x_{0} \in M$ and $u \in C_{0}^{\infty}(M)$. By Theorem 2.1, we have $d_{x_{0}} \Delta_{g} d_{x_{0}} \geq n-1$. Consequently,

$$
\begin{aligned}
\int_{M} \frac{|u|^{p}}{d_{x_{0}}^{q}} \mathrm{~d} V_{g} \leq & \frac{1}{n-1} \int_{M} \frac{|u|^{p}}{d_{x_{0}}^{q-1}} \Delta_{g} d_{x_{0}} \mathrm{~d} V_{g} \\
= & -\frac{1}{n-1} \int_{M}\left\langle\nabla_{g}\left(\frac{|u|^{p}}{d_{x_{0}}^{q-1}}\right), \nabla_{g} d_{x_{0}}\right\rangle \mathrm{d} V_{g} \\
= & -\frac{p}{n-1} \int_{M} \frac{|u|^{p-2} u}{d_{x_{0}}^{q-1}}\left\langle\nabla_{g}|u|, \nabla_{g} d_{x_{0}}\right\rangle \mathrm{d} V_{g} \\
& +\frac{q-1}{n-1} \int_{M} \frac{|u|^{p}}{d_{x_{0}}^{q}}\left|\nabla_{g} d_{x_{0}}\right|^{2} \mathrm{~d} V_{g} .
\end{aligned}
$$

Since $\left|\nabla_{g} d_{x_{0}}\right|=1$, a reorganization of the above estimate implies that

$$
\frac{n-q}{p} \int_{M} \frac{|u|^{p}}{d_{x_{0}}^{q}} \mathrm{~d} V_{g} \leq-\int_{M} \frac{|u|^{p-2} u}{d_{x_{0}}^{q-1}}\left\langle\nabla_{g}|u|, \nabla_{g} d_{x_{0}}\right\rangle \mathrm{d} V_{g} \leq \int_{M} \frac{|u|^{p-2} u}{d_{x_{0}}^{q-1}}\left|\nabla_{g} u\right| \mathrm{d} V_{g}
$$

By applying the Schwartz inequality, it yields the desired inequality $(\mathbf{C K N})_{x_{0}}$.

The proof of the sharpness of $\frac{(n-q)^{2}}{p^{2}}$ in $(\mathbf{C K N})_{x_{0}}$ works in a similar manner as in Theorem 1.1, by exploiting the fact that in the Euclidean setting the inequality $(\mathbf{C K N})_{x_{0}}$ has the form

$$
\left(\int_{\mathbb{R}^{n}}|\nabla u|^{2} \mathrm{~d} x\right)\left(\int_{\mathbb{R}^{n}} \frac{|u|^{2 p-2}}{|x|^{2 q-2}} \mathrm{~d} x\right) \geq \frac{(n-q)^{2}}{p^{2}}\left(\int_{\mathbb{R}^{n}} \frac{|u|^{p}}{|x|^{q}} \mathrm{~d} x\right)^{2}, \forall u \in C_{0}^{\infty}\left(\mathbb{R}^{n}\right),
$$

which has a class of positive extremals given in (1.3).

(ii) $($ a $) \Rightarrow\left(\right.$ c). According to the hypothesis, $\frac{(n-q)^{2}}{p^{2}}$ is sharp and there exists a positive extremal function $w_{0}$ in $(\mathbf{C K N})_{x_{0}}$ for some $x_{0} \in M$. In particular, in relation (4.1) we should have the equality

$$
\int_{M} \frac{w_{0}^{p}}{d_{x_{0}}^{q}} \mathrm{~d} V_{g}=\frac{1}{n-1} \int_{M} \frac{w_{0}^{p}}{d_{x_{0}}^{q-1}} \Delta_{g} d_{x_{0}} \mathrm{~d} V_{g}
$$

Since $w_{0}>0$ and $d_{x_{0}} \Delta_{g} d_{x_{0}} \geq n-1$, relation (4.2) implies that we necessarily have $d_{x_{0}} \Delta_{g} d_{x_{0}}=n-1$, thus $\Delta_{g}\left(d_{x_{0}}^{2}\right)=2 n$. The rest of the proof is similar to that of Theorem 1.1 .

4.2. Hardy-Poincaré inequality: proof of Theorem 1.4. Before to prove Theorem 1.4, we present a quantitative version of the Hardy-Poincaré inequality on CartanHadamard manifolds. 
Theorem 4.1. [Quantitative Hardy-Poincaré inequality] Let $(M, g)$ be an $n$-dimensional $(n \geq 3)$ Cartan-Hadamard manifold with sectional curvature bounded from above by $c \leq 0$. Then for every $x_{0} \in M$ and $u \in C_{0}^{\infty}(M)$ we have

$$
\int_{M}\left|\nabla_{g} u\right|^{2} \mathrm{~d} V_{g} \geq \frac{(n-2)^{2}}{4} \int_{M}\left(1+\frac{2(n-1)}{n-2} \mathbf{D}_{c}\left(d_{x_{0}}\right)\right) \frac{u^{2}}{d_{x_{0}}^{2}} \mathrm{~d} V_{g} .
$$

In addition, the constant $\frac{(n-2)^{2}}{4}$ is sharp and never achieved.

Proof. Let $x_{0} \in M$ and $u \in C_{0}^{\infty}(M)$ be arbitrarily and fix $\gamma=\frac{n-2}{2}>0$. We consider the function $v=d_{x_{0}}^{\gamma} u$. Thus, for $u=d_{x_{0}}^{-\gamma} v$ one has

$$
\nabla_{g} u=-\gamma d_{x_{0}}^{-\gamma-1} v \nabla_{g} d_{x_{0}}+d_{x_{0}}^{-\gamma} \nabla_{g} v
$$

Therefore, it yields

$$
\left|\nabla_{g} u\right|^{2} \geq \gamma^{2} d_{x_{0}}^{-2 \gamma-2} v^{2}\left|\nabla_{g} d_{x_{0}}\right|^{2}-2 \gamma d_{x_{0}}^{-2 \gamma-1} v\left\langle\nabla_{g} d_{x_{0}}, \nabla_{g} v\right\rangle
$$

Since $\left|\nabla_{g} d_{x_{0}}\right|=1$ a.e. on $M$, after integrating the latter inequality, we obtain

$$
\int_{M}\left|\nabla_{g} u\right|^{2} \mathrm{~d} V_{g} \geq \gamma^{2} \int_{M} d_{x_{0}}^{-2 \gamma-2} v^{2} \mathrm{~d} V_{g}+R_{0}
$$

where

$$
\begin{aligned}
R_{0} & =-2 \gamma \int_{M} d_{x_{0}}^{-2 \gamma-1} v\left\langle\nabla_{g} d_{x_{0}}, \nabla_{g} v\right\rangle \mathrm{d} V_{g}=\frac{1}{2} \int_{M}\left\langle\nabla_{g}\left(v^{2}\right), \nabla_{g}\left(d_{x_{0}}^{-2 \gamma}\right)\right\rangle \mathrm{d} V_{g} \\
& =-\frac{1}{2} \int_{M} v^{2} \Delta_{g}\left(d_{x_{0}}^{-2 \gamma}\right) \mathrm{d} V_{g} \\
& =\gamma \int_{M} v^{2} d_{x_{0}}^{-2 \gamma-2}\left(-2 \gamma-1+d_{x_{0}} \Delta_{g} d_{x_{0}}\right) \mathrm{d} V_{g} \\
& \geq \frac{(n-1)(n-2)}{2} \int_{M}\left(d_{x_{0}} \mathbf{c t}_{c}\left(d_{x_{0}}\right)-1\right) \frac{u(x)^{2}}{d_{x_{0}}^{2}} \mathrm{~d} V_{g}, \quad \text { (see Theorem 2.1) } \\
& =\frac{(n-1)(n-2)}{2} \int_{M} \mathbf{D}_{c}\left(d_{x_{0}}\right) \frac{u(x)^{2}}{d_{x_{0}}^{2}} \mathrm{~d} V_{g},
\end{aligned}
$$

which completes the first part of the proof.

We shall prove in the sequel that $\gamma^{2}=\frac{(n-2)^{2}}{4}$ is sharp in $(\mathbf{H P})_{x_{0}}$, i.e.,

$$
\frac{(n-2)^{2}}{4}=\inf _{u \in C_{0}^{\infty}(M) \backslash\{0\}} \frac{\int_{M}\left|\nabla_{g} u\right|^{2} \mathrm{~d} V_{g}}{\int_{M}\left(1+\frac{2(n-1)}{n-2} \mathbf{D}_{c}\left(d_{x_{0}}\right)\right) \frac{u^{2}}{d_{x_{0}}^{2}} \mathrm{~d} V_{g}} .
$$

Fix the numbers $R>r>0$ and a smooth cutoff function $\psi: M \rightarrow[0,1]$ with $\operatorname{supp}(\psi)=$ $\overline{B\left(x_{0}, R\right)}$ and $\psi(x)=1$ for $x \in B\left(x_{0}, r\right)$, and for every $\varepsilon>0$, let

$$
u_{\varepsilon}=\left(\max \left\{\varepsilon, d_{x_{0}}\right\}\right)^{-\gamma} \text {. }
$$

On one hand,

$$
\begin{aligned}
I_{1}(\varepsilon) & :=\int_{M}\left|\nabla_{g}\left(\psi u_{\varepsilon}\right)\right|^{2} \mathrm{~d} V_{g} \\
& =\int_{B\left(x_{0}, r\right)}\left|\nabla_{g}\left(\psi u_{\varepsilon}\right)\right|^{2} \mathrm{~d} V_{g}+\int_{B\left(x_{0}, R\right) \backslash B\left(x_{0}, r\right)}\left|\nabla_{g}\left(\psi u_{\varepsilon}\right)\right|^{2} \mathrm{~d} V_{g} \\
& =\gamma^{2} \int_{B\left(x_{0}, r\right) \backslash B\left(x_{0}, \varepsilon\right)} d_{x_{0}}^{-2 \gamma-2} \mathrm{~d} V_{g}+\tilde{I}_{1}(\varepsilon),
\end{aligned}
$$


where the quantity

$$
\tilde{I}_{1}(\varepsilon)=\int_{B\left(x_{0}, R\right) \backslash B\left(x_{0}, r\right)}\left|\nabla_{g}\left(\psi u_{\varepsilon}\right)\right|^{2} \mathrm{~d} V_{g}
$$

is finite and does not depend on $\varepsilon>0$ whenever $\varepsilon<r$. On the other hand,

$$
\begin{aligned}
I_{2}(\varepsilon) & :=\int_{M}\left(1+\frac{2(n-1)}{n-2} \mathbf{D}_{c}\left(d_{x_{0}}\right)\right) \frac{\left(\psi u_{\varepsilon}\right)^{2}}{d_{x_{0}}^{2}} \mathrm{~d} V_{g} \\
& \geq \int_{M} \frac{\left(\psi u_{\varepsilon}\right)^{2}}{d_{x_{0}}^{2}} \mathrm{~d} V_{g} \\
& \geq \int_{B\left(x_{0}, r\right) \backslash B\left(x_{0}, \varepsilon\right)} d_{x_{0}}^{-2 \gamma-2} \mathrm{~d} V_{g}=: \tilde{I}_{2}(\varepsilon) .
\end{aligned}
$$

By applying the layer cake representation, we deduce that for $0<\varepsilon<r$, one has

$$
\begin{aligned}
\tilde{I}_{2}(\varepsilon) & =\int_{B\left(x_{0}, r\right) \backslash B\left(x_{0}, \varepsilon\right)} d_{x_{0}}^{-2 \gamma-2} \mathrm{~d} V_{g}=\int_{B\left(x_{0}, r\right) \backslash B\left(x_{0}, \varepsilon\right)} d_{x_{0}}^{-n} \mathrm{~d} V_{g} \\
& \geq \int_{r^{-n}}^{\varepsilon^{-n}} \operatorname{Vol}_{g}\left(B\left(x_{0}, \rho^{-\frac{1}{n}}\right)\right) \mathrm{d} \rho \\
& \geq \omega_{n} \int_{r^{-n}}^{\varepsilon^{-n}} \rho^{-1} \mathrm{~d} \rho \\
& =n \omega_{n}(\ln r-\ln \varepsilon) .
\end{aligned}
$$

In particular, $\lim _{\varepsilon \rightarrow 0^{+}} \tilde{I}_{2}(\varepsilon)=+\infty$. Thus, from the above relations it follows that

$$
\begin{aligned}
\frac{(n-2)^{2}}{4} & \leq \inf _{u \in C_{0}^{\infty}(M) \backslash\{0\}} \frac{\int_{M}\left|\nabla_{g} u\right|^{2} \mathrm{~d} V_{g}}{\int_{M}\left(1+\frac{2(n-1)}{n-2} \mathbf{D}_{c}\left(d_{x_{0}}\right)\right) \frac{u^{2}}{d_{x_{0}}^{2}} \mathrm{~d} V_{g}} \\
& \leq \lim _{\varepsilon \rightarrow 0^{+}} \frac{I_{1}(\varepsilon)}{I_{2}(\varepsilon)} \leq \lim _{\varepsilon \rightarrow 0^{+}} \frac{\gamma^{2} \tilde{I}_{2}(\varepsilon)+\tilde{I}_{1}(\varepsilon)}{\tilde{I}_{2}(\varepsilon)} \\
& =\gamma^{2}=\frac{(n-2)^{2}}{4},
\end{aligned}
$$

which concludes the proof of (4.4).

If we assume the function $u_{0} \neq 0$ is an extremal in $(\mathbf{H P})_{x_{0}}$, on one hand, due to (4.3) we have

$$
\int_{M} d_{x_{0}}^{-2 \gamma}\left|\nabla_{g} v_{0}\right|^{2} \mathrm{~d} V_{g}=0
$$

where $v_{0}=d_{x_{0}}^{\gamma} u_{0}$. By (4.6) it follows that $v_{0}$ is a constant function, thus $u_{0}=c_{0} d_{x_{0}}^{-\gamma}$ for some $c_{0} \in \mathbb{R} \backslash\{0\}$. On the other hand, similar estimates as above show that

$$
\int_{M}\left|\nabla_{g} u_{0}\right|^{2} \mathrm{~d} V_{g}=\gamma^{2} \int_{M} \frac{u_{0}^{2}}{d_{x_{0}}^{2}} \mathrm{~d} V_{g}=c_{0}^{2} \gamma^{2} \int_{M} d_{x_{0}}^{-n} \mathrm{~d} V_{g}=+\infty,
$$

i.e., $u_{0} \notin H^{1}\left(M, \mathrm{~d} V_{g}\right)$ and $\frac{u_{0}}{d_{x_{0}}} \notin L^{2}\left(M, \mathrm{~d} V_{g}\right)$, a contradiction.

Proof of Theorem 1.4. By the continued fraction representation of the function $\rho \mapsto$ $\operatorname{coth}(\rho)$, one has

$$
\rho \operatorname{coth}(\rho)-1 \geq \frac{3 \rho^{2}}{\pi^{2}+\rho^{2}} \text { for all } \rho>0 .
$$

Now, the inequality follows at once from this estimate and Theorem 4.1. 
Remark 4.1. (i) Our arguments work also for weighted Hardy-Poincaré inequalities; for simplicity, we presented $(\mathbf{H P})_{x_{0}}$ in its simplest form.

(ii) Kombe and Özaydin [28, 29] investigated the sharp constant in the Hardy-Poincaré inequality on the hyperbolic space $\mathbb{H}^{n}, n \geq 3$. As expected, they claimed that

$$
\frac{(n-2)^{2}}{4}=\inf _{u \in C_{0}^{\infty}\left(\mathbb{H}^{n}\right) \backslash\{0\}} \frac{\int_{\mathbb{H}^{n}}\left|\nabla_{\mathbb{H}^{n}} u\right|^{2} \mathrm{~d} V_{\mathbb{H}^{n}}}{\int_{\mathbb{H}^{n}} \frac{u^{2}}{d^{2}} \mathrm{~d} V_{\mathbb{H}^{n}}},
$$

where the notations come from $\S 3.2$. In order to prove (4.7), the authors used as test functions only those from (4.5) without coupling with an appropriate cutoff function (as in the proof of Theorem 4.1). Although the functions $u_{\varepsilon}$ can be approximated by elements from $C_{0}^{\infty}\left(\mathbb{H}^{n}\right)$, the gap in $[28,29]$ appears due to the fact that $u_{\varepsilon} \notin H^{1}\left(\mathbb{H}^{n}, \mathrm{~d} V_{\mathbb{H}^{n}}\right)$ and $\frac{u_{\varepsilon}}{d} \notin L^{2}\left(\mathbb{H}^{n}, \mathrm{~d} V_{\mathbb{H}^{n}}\right), \varepsilon>0$. Indeed, simple computations show that for every $\varepsilon>0$,

$$
\begin{aligned}
\int_{\mathbb{H}^{n}}\left|\nabla_{\mathbb{H}^{n}} u_{\varepsilon}\right|^{2} \mathrm{~d} V_{\mathbb{H}^{n}} & =(\gamma+\varepsilon)^{2} \int_{\mathbb{H}^{n} \backslash B(0,1)} d^{-2 \gamma-2 \varepsilon-2} \mathrm{~d} V_{\mathbb{H}^{n}} \\
& =(\gamma+\varepsilon)^{2} n \omega_{n} \int_{1}^{\infty} t^{-n-2 \varepsilon}(\sinh t)^{n-1} \mathrm{~d} t=+\infty,
\end{aligned}
$$

and

$$
\int_{\mathbb{H}^{n}} \frac{u_{\varepsilon}^{2}}{d^{2}} \mathrm{~d} V_{\mathbb{H}^{n}} \geq \int_{\mathbb{H}^{n} \backslash B(0,1)} d^{-2 \gamma-2 \varepsilon-2} \mathrm{~d} V_{\mathbb{H}^{n}}=n \omega_{n} \int_{1}^{\infty} t^{-n-2 \varepsilon}(\sinh t)^{n-1} \mathrm{~d} t=+\infty .
$$

(iii) Similar observation as in (ii) has been already made in Yang, Su and Kong [40]. In [40], the authors proved sharp Hardy and Rellich inequalities on Riemannian manifolds with negative sectional curvature. The novelty of our results (Theorem $1.4 \& 4.1$ ) is that improvements appear quantitatively in terms of the sectional curvature.

A similar argument as in Theorem 4.1 leads to the following improvement.

Theorem 4.2. [Double improved Hardy-Poincaré inequality] Let $\Omega$ be a bounded open domain with smooth boundary in an $n$-dimensional $(n \geq 3)$ Cartan-Hadamard manifold with sectional curvature bounded from above by $c \leq 0$. If $x_{0} \in \Omega$ and $R>\sup _{x \in \Omega} d\left(x, x_{0}\right)$, then for all $u \in C_{0}^{\infty}(\Omega)$,

$$
\int_{\Omega}\left|\nabla_{g} u\right|^{2} \mathrm{~d} V_{g} \geq \frac{(n-2)^{2}}{4} \int_{\Omega}\left(1+\frac{2(n-1)}{n-2} \mathbf{D}_{c}\left(d_{x_{0}}\right)\right) \frac{u^{2}}{d_{x_{0}}^{2}} \mathrm{~d} V_{g}+\frac{1}{4} R_{\Omega},
$$

where

$$
R_{\Omega}=\int_{\Omega}\left(1+2(n-1) \ln \left(\frac{e R}{d_{x_{0}}}\right) \mathbf{D}_{c}\left(d_{x_{0}}\right)\right) \frac{u(x)^{2}}{d_{x_{0}}^{2} \ln ^{2}\left(\frac{e R}{d_{x_{0}}}\right)} \mathrm{d} V_{g} .
$$

Proof. Let $x_{0} \in \Omega, u \in C_{0}^{\infty}(\Omega)$ and fix $\gamma=\frac{n-2}{2}>0$. If we consider the function $v=d_{x_{0}}^{\gamma} u$, one has

$$
\left|\nabla_{g} u\right|^{2}=\gamma^{2} d_{x_{0}}^{-2 \gamma-2} v^{2}-2 \gamma d_{x_{0}}^{-2 \gamma-1} v\left\langle\nabla_{g} d_{x_{0}}, \nabla_{g} v\right\rangle+d_{x_{0}}^{-2 \gamma}\left|\nabla_{g} u\right|^{2} .
$$

After an integration over $\Omega$ of the above relation, one can repeat the argument from the proof of Theorem 4.1 to the first two integrands, obtaining

$$
\int_{\Omega}\left|\nabla_{g} u\right|^{2} \mathrm{~d} V_{g} \geq \frac{(n-2)^{2}}{4} \int_{\Omega}\left(1+\frac{2(n-1)}{n-2} \mathbf{D}_{c}\left(d_{x_{0}}\right)\right) \frac{u^{2}}{d_{x_{0}}^{2}} \mathrm{~d} V_{g}+\tilde{R},
$$

where

$$
\tilde{R}=\int_{\Omega} d_{x_{0}}^{-2 \gamma}\left|\nabla_{g} v\right|^{2} \mathrm{~d} V_{g}
$$


Due to the fact that $R>\sup _{x \in \Omega} d\left(x, x_{0}\right)$, the function $h=\ln \frac{e R}{d_{x_{0}}}$ is well defined on $\Omega \backslash\left\{x_{0}\right\}$ and $h \geq 1$. Let $z=h^{-1 / 2} v$. Since

$$
\nabla_{g} v=-\frac{z}{2 d_{x_{0}}} h^{-1 / 2} \nabla_{g} d_{x_{0}}+h^{1 / 2} \nabla_{g} z
$$

it turns out that

$$
\left|\nabla_{g} v\right|^{2} \geq \frac{z^{2}}{4 d_{x_{0}}^{2}} h^{-1}-\frac{z}{d_{x_{0}}}\left\langle\nabla_{g} d_{x_{0}}, \nabla_{g} z\right\rangle
$$

Consequently,

$$
\begin{aligned}
\tilde{R} & =\int_{\Omega} d_{x_{0}}^{-2 \gamma}\left|\nabla_{g} v\right|^{2} \mathrm{~d} V_{g} \\
& \geq \frac{1}{4} \int_{\Omega} d_{x_{0}}^{-2 \gamma-2} h^{-1} z^{2} \mathrm{~d} V_{g}-\frac{1}{2} \int_{\Omega} d_{x_{0}}^{-2 \gamma-1}\left\langle\nabla_{g} d_{x_{0}}, \nabla_{g}\left(z^{2}\right)\right\rangle \mathrm{d} V_{g} \\
& =\frac{1}{4} \int_{\Omega} d_{x_{0}}^{-2} h^{-2} u^{2} \mathrm{~d} V_{g}-\frac{1}{4 \gamma} \int_{\Omega} z^{2} \Delta_{g}\left(d_{x_{0}}^{-2 \gamma}\right) \mathrm{d} V_{g} \\
& =\frac{1}{4} \int_{\Omega} d_{x_{0}}^{-2} h^{-2} u^{2} \mathrm{~d} V_{g}+\frac{1}{2} \int_{\Omega} z^{2} d_{x_{0}}^{-2 \gamma-2}\left(-2 \gamma-1+d_{x_{0}} \Delta_{g} d_{x_{0}}\right) \mathrm{d} V_{g} \\
& \geq \frac{1}{4} \int_{\Omega} d_{x_{0}}^{-2} h^{-2} u^{2} \mathrm{~d} V_{g}+\frac{n-1}{2} \int_{\Omega} \mathbf{D}_{c}\left(d_{x_{0}}\right) d_{x_{0}}^{-2 \gamma-2} z^{2} \mathrm{~d} V_{g} \\
& =\frac{1}{4} \int_{\Omega}\left(1+2(n-1) h \mathbf{D}_{c}\left(d_{x_{0}}\right)\right) \frac{u^{2}}{d_{x_{0}}^{2} h^{2}} \mathrm{~d} V_{g},
\end{aligned}
$$

which concludes the proof.

Remark 4.2. In the limiting case when $c=0\left(\right.$ thus $\mathbf{D}_{c}(\rho)=\mathbf{D}_{0}(\rho)=0$ for every $\left.\rho \geq 0\right)$, the inequality in Theorem 4.2 takes the familiar form

$$
\int_{\Omega}\left|\nabla_{g} u\right|^{2} \mathrm{~d} V_{g} \geq \frac{(n-2)^{2}}{4} \int_{\Omega} \frac{u^{2}}{d_{x_{0}}^{2}} \mathrm{~d} V_{g}+\frac{1}{4} \int_{\Omega} \frac{u^{2}}{d_{x_{0}}^{2} \ln ^{2}\left(\frac{e R}{d_{x_{0}}}\right)} \mathrm{d} V_{g},
$$

see Adimurthi, Chaudhuri and Ramaswamy [1] and Filippas and Tertikas [20] in the Euclidean case, and Kombe and Özaydin [29, Corollary 2.2] in hyperbolic spaces.

Acknowledgment. The author would like to thank the two anonymous reviewers for their suggestions and comments.

\section{REFERENCES}

[1] Adimurthi, N. Chaudhuri, N. Ramaswamy, An improved Hardy Sobolev inequality and its applications. Proc. Amer. Math. Soc. 130 (2002), 489-505.

[2] N. B. Andersen, Hardy's uncertainty principle on hyperbolic spaces. Bull. Austral. Math. Soc. 66 (2002), no. 1, 163-170.

[3] N. B. Andersen, $L^{p}$ versions of Hardy's uncertainty principle on hyperbolic spaces. Proc. Amer. Math. Soc. 131 (2003), no. 9, 2797-2807.

[4] T. Aubin, Meilleures constantes dans le théorème d'inclusion de Sobolev et un théorème de Fredholm non linéaire pour la transformation conforme de la courbure scalaire. J. Funct. Anal. 32 (1979), no. 2, 148-174.

[5] G. Barbatis, S. Filippas, A. Tertikas, A unified approach to improved $L^{p}$ Hardy inequalities with best constants. Trans. Amer. Math. Soc. 356 (2004), 2169-2196.

[6] E. Berchio, L. D’Ambrosio, D. Ganguly, G. Grillo, Improved $L^{p}$-Poincaré inequalities on the hyperbolic space. Nonlinear Anal. 157 (2017), 146-166.

[7] E. Berchio, D. Ganguly, G. Grillo, Sharp Poincar-Hardy and Poincar-Rellich inequalities on the hyperbolic space. J. Funct. Anal. 272 (2017), no. 4, 1661-1703. 
[8] H. Brezis, J. L. Vázquez, Blowup solutions of some nonlinear elliptic problems. Revista Mat. Univ. Complutense Madrid 10 (1997), 443-469.

[9] M. Bridson, A. Haefliger, Metric Spaces of Non-positive Curvature, Fundamental Principles of Mathematical Sciences, 319, Springer, Berlin, 1999.

[10] L. Caffarelli, R. Kohn, L. Nirenberg, First order interpolation inequalities with weight. Compos. Math. 53 (1984), 259-275.

[11] G. Carron, Inégalités de Hardy sur les variétés riemanniennes non-compactes. J. Math. Pures Appl. (9) 76 (1997), no. 10, 883-891.

[12] J. Cheeger, T. Colding, On the structure of spaces with Ricci curvature bounded below I. J. Diff. Geom. 46 (1997), 406-480.

[13] P. Ciatti, F. Ricci, M. Sundari, Heisenberg-Pauli-Weyl uncertainty inequalities and polynomial volume growth. Adv. Math. 215 (2007), no. 2, 616-625.

[14] L. D’Ambrosio, S. Dipierro, Hardy inequalities on Riemannian manifolds and applications. Ann. Inst. H. Poincaré Anal. Non Linéaire 31 (2014), no. 3, 449-475.

[15] M. P. do Carmo, C. Xia, Complete manifolds with non-negative Ricci curvature and the CaffarelliKohn-Nirenberg inequalities. Compos. Math. 140 (2004), 818-826.

[16] O. Druet, E. Hebey, M. Vaugon, Optimal Nash's inequalities on Riemannian manifolds: the influence of geometry. International Mathematics Research Notices, 14, 1999, 735-779.

[17] W. Erb, Uncertainty principles on compact Riemannian manifolds. Appl. Comput. Harmon. Anal. 29 (2010), no. 2, 182-197.

[18] W. Erb, Uncertainty principles on Riemannian manifolds. PhD Dissertation, Technical University Munchen, 2009.

[19] Ch. L. Fefferman, The uncertainty principle. Bull. Amer. Math. Soc. (N.S.) 9 (1983), no. 2, $129-206$.

[20] S. Filippas, A. Tertikas, Optimizing improved Hardy inequalities. J. Funct. Anal. 192 (2002) 186-233.

[21] G. B. Folland, A. Sitaram, The uncertainty principle: a mathematical survey. J. Fourier Anal. Appl. $3(3)(1997) 207-238$.

[22] N. Ghoussoub, A. Moradifam, On the best possible remaining term in the Hardy inequality. Proc. Natl. Acad. Sci. USA 105 (2008), no. 37, 13746-13751.

[23] N. Ghoussoub, A. Moradifam, Bessel pairs and optimal Hardy and Hardy-Rellich inequalities. Math. Ann. 349 (2011), 1-57.

[24] E. Hebey, Nonlinear analysis on manifolds: Sobolev spaces and inequalities. Courant Lecture Notes in Mathematics, 5. New York University, Courant Institute of Mathematical Sciences, New York; American Mathematical Society, Providence, RI, 1999.

[25] W. Heisenberg, Über den anschaulichen Inhalt der quantentheoretischen Kinematik und Mechanik. Z. Physik 43 (1927), 172-198.

[26] J. Jost, Nonpositivity Curvature: Geometric and Analytic Aspects, Birkhäuser, Basel, 1997.

[27] A. Kristály, S. Ohta, Caffarelli-Kohn-Nirenberg inequality on metric measure spaces with applications. Math. Ann. 357 (2013), no. 2, 711-726.

[28] I. Kombe, M. Özaydin, Improved Hardy and Rellich inequalities on Riemannian manifolds. Trans. Amer. Math. Soc. 361 (2009), no. 12, 6191-6203.

[29] I. Kombe, M. Özaydin, Hardy-Poincaré, Rellich and uncertainty principle inequalities on Riemannian manifolds. Trans. Amer. Math. Soc. 365 (2013), no. 10, 5035-5050.

[30] M. Ledoux, Isoperimetry and Gaussian analysis, Lectures on Probability Theory and Statistics (Saint-Flour, 1994), Lecture Notes in Mathematics, Vol. 1648, Springer, Berlin, 1996, pp. 165-294.

[31] P. Li, J. Wang, Weighted Poincaré inequality and rigidity of complete manifolds. Ann. Sci. École Norm. Sup. (4) 39 (2006), no. 6, 921-982.

[32] V. Minerbe, Weighted Sobolev inequalities and Ricci flat manifolds, Geom. Funct. Anal. 18 (2009), no. 5, 1696-1749.

[33] A. R. Nahmod, Generalized uncertainty principles on spaces of homogeneous type. J. Funct. Anal. 119 (1994), no. 1, 171-209.

[34] K. Okoudjou, L. Saloff-Coste, A. Teplyaev, Weak uncertainty principle for fractals, graphs and metric measure spaces. Trans. Amer. Math. Soc. 360 (2008), no. 7, 3857-3873.

[35] Z. Shen, Volume comparison and its applications in Riemann-Finsler geometry. Adv. Math. 128 (1997), no. 2, 306-328.

[36] Z.-Q. Wang, M. Willem, Caffarelli-Kohn-Nirenberg inequalities with remainder terms. J. Funct. Anal. 203 (2003), 550-568.

[37] H. Weyl, The Theory of Groups and Quantum Mechanics. Dover Publications, New York, 1931. 
[38] B. Y. Wu, Y. L. Xin, Comparison theorems in Finsler geometry and their applications. Math. Ann. 337 (2007), no. 1, 177-196.

[39] C. Xia, The Caffarelli-Kohn-Nirenberg inequalities on complete manifolds. Math. Res. Lett. 14 (2007), no. 5, 875-885.

[40] Q. Yang, D. Su, Y. Kong, Hardy inequalities on Riemannian manifolds with negative curvature. Commun. Contemp. Math. 16 (2014), no. 2, 1350043, 24 pp.

[41] W. Zhao, Y. Shen, A universal volume comparison theorem for Finsler manifolds and related results. Canad. J. Math. 65 (2013), no. 6, 1401-1435.

Institute of Applied Mathematics, Óbuda University (formerly Budapest Tech Polytechnical Institution), Budapest, Hungary

Department of Economics, Babeş-Bolyai University, Cluj-Napoca, Romania

E-mail address: alex.kristaly@econ.ubbcluj.ro; alexandrukristaly@yahoo.com 